\title{
A novel supplemental approach to capturing post-marketing safety information on recombinant factor Vlla in acquired hemophilia: the Acquired Hemophilia Surveillance project
}

\author{
This article was published in the following Dove Press journal: \\ Journal of Blood Medicine \\ 13 January 2014 \\ Number of times this article has been viewed
}

\author{
Steven R Lentz' \\ Anand Tandra ${ }^{2}$ \\ Robert Z Gut ${ }^{3}$ \\ David L Cooper ${ }^{3}$ \\ 'Division of Hematology, \\ Oncology and Blood and Marrow \\ Transplantation, Department of \\ Internal Medicine, University of lowa, \\ lowa City, IA, USA; ${ }^{2} \mathrm{Hematology,}$ \\ Indiana Hemophilia and Thrombosis \\ Center, Indianapolis, IN, USA; ${ }^{3}$ Clinical, \\ Medical, and Regulatory Affairs, Novo \\ Nordisk, Inc, Princeton, NJ, USA
}

\section{Dear editor}

Acquired hemophilia (AH) is a rare (incidence is 1 per 1.5 million) but often severe bleeding disorder characterized by autoantibodies to coagulation factor VIII (FVIII). It is associated with life-threatening bleeding and $\sim 20 \%$ mortality. ${ }^{1,2}$ Recombinant factor VIIa (rFVIIa; NovoSeven ${ }^{\circledR}$ RT, Novo Nordisk A/S, Bagsværd, Denmark) received an indication from the US Food and Drug Administration (FDA) in October 2006 for the treatment of bleeding episodes and the prevention of bleeding in surgical interventions or invasive procedures in patients with $\mathrm{AH} .{ }^{3}$

The approval of rFVIIa for AH was supported by data collected through the Hemostasis and Thrombosis Research Society (HTRS) Registry since the product's original approval in 1999. The HTRS Registry is a longitudinal US database created by the Society in collaboration with Novo Nordisk to study treatment strategies for patients with bleeding disorders and to provide post-marketing surveillance. It was adapted in 2006 to fulfill a 5 -year commitment by Novo Nordisk to the FDA to provide surveillance around AH.

AH affects only about 350 patients per year in the US and many patients are treated outside of the federally designated and funded hemophilia treatment center (HTC) network by hematologists, hematologist/oncologists, and other hospital-based physicians. Many hospitals and most of the $\sim 140$ HTCs do not frequently see patients with this disorder, or else see them so rarely that maintaining active IRB renewals and trained staff to participate in the HTRS Registry over 5 years would be considered burdensome. In addition, requests for consultation on such patients from those who first see the patients (emergency medicine, hospitalists, critical care, obstetrics/gynecology, rheumatology) may originate within the hematology staff at any hospital and may be limited to phone consultation with a remote hematologist, thus limiting the amount of data available to the hematologist regarding patient treatment and outcomes.

The objective of the Acquired Hemophilia Surveillance (AHS) project was to implement a simple IRB-exempt case report surveillance system to document the at-risk population exposed to rFVIIa and to capture data on the incidence of adverse events (AEs), particularly thromboembolic events (TEs) in these patients. It was specifically targeted to healthcare professionals (HCPs), including non-hematologists who did not belong to HTRS or who were based at HTCs that were resource constrained from considering HTRS participation.
Correspondence: Steven R Lentz Division of Hematology, Oncology and Blood and Marrow Transplantation, Department of Internal Medicine, C32 $\mathrm{GH}$, University of lowa, 200 Hawkins Drive, lowa City, IA 52242, USA

Tel +l 3193564048

Fax +l 3193538383

Email steven-lentz@uiowa.edu 
This AHS system was launched in April 2008 as a onepage fax-in reporting system maintained by Novo Nordisk. In June 2008, it was converted to a secured web-based platform located at novosevensurveillance.com by Outcome Sciences (Cambridge, MA, USA), a third-party contract research organization that also entered the preliminary faxed-in reports and maintained the database. The AHS web portal allowed for simple electronic entry of demographic and treatment information on acquired hemophilia cases, with the addition of a full safety report form where applicable. AHS takes advantage of the HIPAA exclusion for collection of safety information (45 CFR §164.512(b)(iii)(D)). Reporting HCPs were provided fair market compensation per case entry.

Field-based medical liaisons were integrally involved in obtaining information about potential $\mathrm{AH}$ cases from visits to HTC and non-HTC sites where they discussed both the HTRS Registry (primary goal) and the AHS project (secondary goal) with HCPs as a means of contributing AH information for post-marketing safety. Medical liaisons distributed copies of a "dear doctor" letter describing the AH post-marketing safety requirement and the AHS project, magnets with the HTRS and AHS project contact numbers, and other supporting materials.

Bleed data collected from AHS between April 2008 and 30 November 2011 were analyzed for the safety of rFVIIa treatment in patients with $\mathrm{AH}$. Information on demographics, rFVIIa dosing (entered via an optional free text field) and incidence of thrombotic events was collected and reported using descriptive statistics. Reporting HCPs were required to complete an affirmation about presence/absence of adverse events, and if reporting adverse events to complete a detailed safety information form consistent with the data required to complete a standard MedWatch report.

From April 2008 through 30 November 2011, 38 individual HCPs submitted 99 case reports (including 65 treated with rFVIIa) via facsimile or electronic data capture interface. The reports were from both HTCs (44) and non-HTCs (48), and more commonly from centers that did not participate in HTRS (84) than did participate (8).

Of the 99 patients, 41 (41\%) were male and $58(59 \%)$ were female. The mean age was 66.6 years (range 16.4 to 97.3 years). The most common underlying conditions were autoimmune (34 patients), malignancy (12 patients), and post-partum (5 patients).

The mean (SD) anti-VIII titer was 154.5 (453.7) Bethesda units (BU) and the median (range) anti-VIII titer was $35(1-3,789)$ BU. Factor VIII levels were reported for 93 of the 99 patients. Fifty-eight percent (57/99) of the subjects had reported factor VIII levels $\leq 1 \%$. The mean (median) factor VIII level in 36 remaining patients ( $>1 \%$ FVIII levels) was $8.1 \%(4.0 \%)$ with a range of $1.8 \%-50 \%$. The mean (median) reported discrete bleeding episodes per patient was 4.3 (2.0) with a range of $0-100$ episodes since the time of AH diagnosis. Most of these episodes were spontaneous (84 patients), surgical (14 patients), or related to a procedure (12 patients). Details on specific bleeding episodes were not captured in this brief report format.

The use of rFVIIa was indicated in 65 case reports $(83 \%)$, including 50 first-line treated cases (77\%), 14 second-line treated cases $(22 \%)$, and one with unspecified line of treatment. A bypassing agent other than rFVIIa was indicated to have been used in 13 case reports (17\%). No bypassing agent was indicated to have been used in 21 case reports.

The acquired hemophilia condition was reported to have been resolved in 51 cases (52\%) and not resolved in 30 cases (30\%). Resolution was reported as "unsure" in 17 cases and not reported for one case. The mean (median) time to acquired hemophilia resolution was 7.5 (2.0) months with a range of 1-52 months. There were five reported deaths. There were 13 cases for which the mortality status was reported as "unsure." In the 65 cases where rFVIIa was used, the reporters affirmed that none of the patients suffered an adverse event that was considered possibly/probably related to rFVIIa. Therefore, there was no detailed treatment and outcome information requested or provided on adverse events.

Reports of dosing as a write-in field, most with limited detail, suggest that full treatment records were not available or reviewed during the preparation of many of these reports, and some patients might have been treated at facilities outside the reporting sites. Since many of the reports also reflect that patients experienced multiple episodes (mean 4.3 per patient), the interpretation of general information provided in response to a single question on efficacy at 24 and 72 hours remains unclear. Among the 50 patients who received rFVIIa as firstline treatment and had outcomes reported, 47 and 46 were reported to have "excellent/good" or "fair/partially effective" outcome at 24 and 72 hours. Among the 14 patients who received rFVIIa as second-line treatment and had outcomes reported, 12 were reported to have an "excellent/good" or "fair/partially effective" outcome at 24 and 72 hours.

For rare disorders or rare complications of common disorders, the standard post-marketing surveillance approach of an IRB-approved observational or product registry may have significant limitations and barriers at the site-implementation level. AHS provides an innovative approach to augment data from formal post-marketing registries under a HIPAA 
exclusion for HTCs, hematology/oncology practices, and even primary hospital-based treating physicians/pharmacists (emergency room, intensive care unit, post-partum unit) to capture basic surveillance data for $\mathrm{AH}$ patients exposed to rFVIIa. AHS was not designed to provide registry-level data, but rather to capture $25 \%$ to $50 \%$ of identified case reports where registry data entry would not occur. Medical liaisons can play an important role in the identification of patients with rare disorders or complications and can provide information about the primary PMS registry and/or backup systems.

The AHS project reaffirms the safety of rFVIIa and the low rate of thrombotic complications. However, AHS lacks the detailed information available through traditional research registries at large centers, such as data on individual bleeds, treatment of individual bleeding episodes, rFVIIa dosing, time to hemostasis, and the relationship of immunosuppressive treatments to $\mathrm{AH}$ resolution.

\section{Acknowledgments}

The authors acknowledge the assistance of Outcome Sciences, who managed this project and managed the data and analysis under funding from Novo Nordisk Inc, and Abha Chandra from Novo Nordisk for analytical and editorial assistance.

\section{Disclosure}

RZG and DLC are employees of Novo Nordisk Inc, the company that markets rFVIIa and is responsible for postmarketing surveillance of its use in acquired hemophilia. SRL is an investigator and steering committee member for Novo Nordisk in other unrelated trials. SRL and AT received per-case fees to their institutions for entry of data during the AHS project. No authors received any remuneration for the preparation and submission of this letter.

\section{References}

1. Collins PW, Hirsch S, Baglin TP, et al. UK Haemophilia Doctors' Organisation. Acquired hemophilia A in the UK: A two year national surveillance study by UK Haemophilia Centre Doctors Organization. Blood. 2007;109:1870-1877.

2. Green D, Lechner K. A survey of 215 non-hemophilia patients with inhibitors to factor VIII. Thrombo Haemost. 1981;45:200-203.

3. NovoSeven RT (Coagulation Factor VIIa [Recombinant], Room Temperature Stable) [prescribing information]. Bagsvaerd, Denmark: Novo Nordisk A/S, 2010.
Journal of Blood Medicine

\section{Publish your work in this journal}

The Journal of Blood Medicine is an international, peer-reviewed, open access, online journal publishing laboratory, experimental and clinical aspects of all topics pertaining to blood based medicine including but not limited to: Transfusion Medicine; Blood collection, Donor issues, Transmittable diseases, and Blood banking logistics; Immunohematology; Artificial and alternative

\section{Dovepress}

blood based therapeutics; Hematology; Biotechnology/nanotechnology of blood related medicine; Legal aspects of blood medicine; Historical perspectives. The manuscript management system is completely online and includes a very quick and fair peer-review system. Visit http://www.dovepress.com/ testimonials.php to read real quotes from published authors. 